\title{
Near-infrared Stellar Populations in the Metal-poor, Dwarf Irregular Galaxies Sextans A and Leo A
}

DOI:

10.3847/1538-4357/aaa542

\section{Document Version}

Final published version

Link to publication record in Manchester Research Explorer

\section{Citation for published version (APA):}

Jones, O. C., Maclay, M. T., Boyer, M. L., Meixner, M., McDonald, I., \& Meskhidze, H. (2018). Near-infrared Stellar Populations in the Metal-poor, Dwarf Irregular Galaxies Sextans A and Leo A. Astrophysical Journal, 854(2), [117]. https://doi.org/10.3847/1538-4357/aaa542

\section{Published in:}

Astrophysical Journal

\section{Citing this paper}

Please note that where the full-text provided on Manchester Research Explorer is the Author Accepted Manuscript or Proof version this may differ from the final Published version. If citing, it is advised that you check and use the publisher's definitive version.

\section{General rights}

Copyright and moral rights for the publications made accessible in the Research Explorer are retained by the authors and/or other copyright owners and it is a condition of accessing publications that users recognise and abide by the legal requirements associated with these rights.

\section{Takedown policy}

If you believe that this document breaches copyright please refer to the University of Manchester's Takedown Procedures [http://man.ac.uk/04Y6Bo] or contact uml.scholarlycommunications@manchester.ac.uk providing relevant details, so we can investigate your claim.

\section{OPEN ACCESS}




\title{
Near-infrared Stellar Populations in the Metal-poor, Dwarf Irregular Galaxies Sextans A and Leo A
}

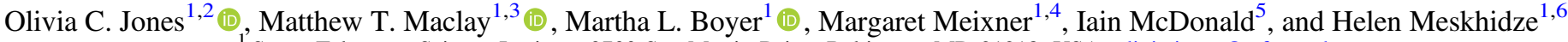 \\ 'Space Telescope Science Institute, 3700 San Martin Drive, Baltimore, MD 21218, USA; olivia.jones@stfc.ac.uk \\ ${ }^{2}$ UK Astronomy Technology Centre, Royal Observatory, Blackford Hill, Edinburgh, EH9 3HJ, UK \\ ${ }^{3}$ Carleton College, 1 North College Street, Northfield, MN 55057, USA \\ ${ }^{4}$ The Johns Hopkins University, Department of Physics and Astronomy, 366 Bloomberg Center, 3400 N. Charles Street, Baltimore, MD 21218, USA \\ Jodrell Bank Centre for Astrophysics, Alan Turing Building, School of Physics and Astronomy, The University of Manchester, \\ Oxford Road, Manchester, M13 9PL, UK \\ ${ }^{6}$ Elon University, 100 Campus Drive, Elon, NC 27244, USA \\ Received 2017 September 14; revised 2017 December 8; accepted 2017 December 18; published 2018 February 16
}

\begin{abstract}
We present $J H K_{s}$ observations of the metal-poor $([\mathrm{Fe} / \mathrm{H}]<-1.40)$ dwarf-irregular galaxies, Leo A and Sextans A, obtained with the WIYN High-resolution Infrared Camera at Kitt Peak. Their near-IR stellar populations are characterized by using a combination of color-magnitude diagrams and by identifying long-period variable stars. We detected red giant and asymptotic giant branch stars, consistent with membership of the galaxy's intermediateage populations (2-8 Gyr old). Matching our data to broadband optical and mid-IR photometry, we determine luminosities, temperatures, and dust-production rates (DPR) for each star. We identify 32 stars in Leo A and 101 stars in Sextans A with a DPR $>10^{-11} M_{\odot} \mathrm{yr}^{-1}$, confirming that metal-poor stars can form substantial amounts of dust. We also find tentative evidence for oxygen-rich dust formation at low metallicity, contradicting previous models that suggest oxygen-rich dust production is inhibited in metal-poor environments. The total rates of dust injection into the interstellar medium of Leo A and Sextans A are $(8.2 \pm 1.8) \times 10^{-9} M_{\odot} \mathrm{yr}^{-1}$ and $(6.2 \pm 0.2) \times 10^{-7} M_{\odot} \mathrm{yr}^{-1}$, respectively. The majority of this dust is produced by a few very dusty evolved stars and does not vary strongly with metallicity.
\end{abstract}

Key words: circumstellar matter - infrared: stars - stars: late-type - stars: mass-loss

Supporting material: machine-readable tables

\section{Introduction}

Toward the end of their nuclear-burning lives, stars of initial mass $1 M_{\odot} \lesssim M \lesssim 8 M_{\odot}$ evolve along the asymptotic giant branch (AGB). During this short evolutionary phase ( $t \sim 0.5-10 \mathrm{Myr}$; Vassiliadis \& Wood 1993; Girardi \& Marigo 2007), AGB stars are among the most luminous infrared (IR) sources in a galaxy (Maraston et al. 2006; Melbourne et al. 2012), they thermally pulsate and experience significant mass loss of up to $10^{-4} M_{\odot} \mathrm{yr}^{-1}$. Mass-loss from AGB stars increases as the star evolves. This gas and dust chemically enriches the interstellar medium (ISM).

During the thermally pulsating AGB (TP-AGB) phase, the stellar atmosphere can transition from oxygen rich to carbon rich via the dredge-up process from the stellar core (Iben \& Renzini 1983). Oxygen-rich AGB stars have an atmospheric $\mathrm{C} / \mathrm{O}<1$, while carbon stars have $\mathrm{C} / \mathrm{O}>1$. This transition depends on the star's initial mass and metallicity. At solar metallicity, stars with an initial mass of $\sim 2-4 M_{\odot}$ form carbon stars (Marigo \& Girardi 2007; Ventura \& Marigo 2010). At lower metallicities, there is less initial oxygen available, thus fewer dredge-up events are required to form carbon stars, resulting in a wider range of progenitor masses (Ferrarotti \& Gail 2001; Di Criscienzo et al. 2013). This has a significant effect on the stars' IR colors. Near-IR wavelengths, where the star emits the majority of its flux, can be used to investigate a galaxy's intermediate-age population, which can be used to trace their baryonic enrichment of the host galaxy.

Metal-poor $(-2.1 \lesssim[\mathrm{Fe} / \mathrm{H}] \lesssim-1.1)$ dust-producing AGB stars have been observed in the mid-IR by the DUST in the Nearby Galaxies with The Spitzer (DUSTiNGS) survey (Boyer et al. 2015b, 2015c). This survey used variability criteria to identify luminous AGB stars in 50 dwarf galaxies within 1.5 Mpc. These galaxies offer a fantastic opportunity to investigate evolved stellar populations over a wide range of environments, star formation histories (SFH) and metallicity. In this work, we focus on the near-IR population of two galaxies in the DUSTiNGS survey, Sextans A and Leo A. Their properties are listed in Table 1.

Sextans A (DDO 75) is an extremely metal-poor $([\mathrm{Fe} / \mathrm{H}] \sim-1.85$ to -1.40 ; Dolphin et al. 2003b), dwarf irregular galaxy. Located at a distance of $1.45 \mathrm{Mpc}$ (McConnachie 2012), it is a member of the NGC 3109 association (van den Bergh 2000), which may or may not be bound to the Local Group. Photometric studies of Sextans A reveal that the galaxy contains an old stellar population (which formed 10-14 Gyr ago), and a younger blue population, which formed during the past $2.5 \mathrm{Gyr}$ (Dohm-Palmer et al. 2002). The star formation rate (SFR) of Sextans A has increased dramatically in the past few gigayears; from a quiescent period between 2.5 and $10 \mathrm{Gyr}$ ago, to a sudden burst in star formation starting 1-2.5 Gyr ago, with another considerable increase over the past 0.06 Gyr (Dolphin et al. 2003b; Weisz et al. 2014; Camacho et al. 2016). There has been little chemical enrichment within Sextans A over the last 10 Gyr: Sextans A is one of the lowest-metallicity objects in which variable AGB stars have been detected (Boyer et al. 2015c; McQuinn et al. 2017). The galaxy also contains a planetary nebula (Magrini et al. 2005) and several short-period Cepheids (Dolphin et al. 2003a). 
Table 1

Sextans A and Leo A Parameters Adopted in This Work ${ }^{\mathrm{a}}$

\begin{tabular}{lcc}
\hline \hline Parameter & Sextans A & Leo A \\
\hline R.A. & $10: 11: 00.8$ & $09: 59: 26.5$ \\
Decl. & $-04: 41: 34$ & $+30: 44: 47$ \\
Distance, $d(\mathrm{kpc})$ & $1432 \pm 53$ & $789 \pm 44$ \\
$(m-M)_{0}(\mathrm{mag})$ & $25.6 \pm 0.1$ & $24.51 \pm 0.12$ \\
{$[\mathrm{Fe} / \mathrm{H}]$} & -1.85 & $-1.4^{\mathrm{b}}$ \\
Half-light Radius $\left(^{\prime}\right)$ & 2.47 & 2.15 \\
Stellar Mass $\left(10^{6} M_{\odot}\right)$ & 44 & 6 \\
$E(B-V)(\operatorname{mag})$ & 0.045 & 0.021 \\
$I$ TRGB (mag) & 21.76 & 20.5 \\
\hline
\end{tabular}

Notes.

${ }^{\mathrm{a}}$ The fundamental parameters of the Sextans A and Leo A galaxies are taken from McConnachie (2012), unless otherwise noted.

${ }^{\mathrm{b}}$ Kirby et al. (2017).

Leo A (also named Leo III or DDO 69) is an isolated, dwarf irregular galaxy in the Local Group, at a distance of about $790 \mathrm{kpc}$ (Dolphin et al. 2002; Bernard et al. 2013). This gasrich galaxy (Young \& Lo 1996) contains both young and old populations (Cole et al. 2007) and is thought to be a darkmatter-dominated $(>80 \%)$ stellar system (Brown et al. 2007). Leo $\mathrm{A}$ is metal-poor $([\mathrm{Fe} / \mathrm{H}] \sim-2.1$ to -1.2 ; Kirby et al. 2017), and the majority of its stellar population is of a young to intermediate age, forming within the last 5-8 Gyr. The last major episode of star formation occurred around $3 \mathrm{Gyr}$ ago. Ongoing star formation activity is traced by three H II, which are found in the galaxy (Strobel et al. 1991; Weisz et al. 2014). While the detection of RR Lyr stars by Dolphin et al. (2002) indicates that an ancient stellar population formed $>11 \mathrm{Gyr}$ ago, possibly during the era of reionization. Furthermore, five variable AGB candidates were identified by Boyer et al. (2015c) and a planetary nebula was discovered by Magrini et al. (2003).

The goal of this paper is to identify the dusty evolved stellar populations in metal-poor irregular galaxies, Sextans A and Leo A. This paper is organized as follows. In Section 2, we describe the WHIRC observations and data reduction. The photometric results and color-magnitude diagrams are presented in Section 3. We analyze these results in Section 4 and compare these findings with other studies. Section 5 contains a summary and conclusions.

\section{Observations and Data Reduction}

\subsection{Observations}

Ground-based observations of Sextans A and Leo A were obtained through the broadband $J H K_{s}$ filters using the WIYN High-resolution Infrared Camera (WHIRC; Meixner et al. 2010) mounted on the $3.5 \mathrm{~m}$ WIYN telescope at the Kitt Peak National Observatory. The central wavelengths and bandwidths of the three filters can be found in Table 2 . The WHIRC camera field of view is $3 ! 4 \times 3 ! 4$ with a plate scale of $\approx 0$ ". 1 pixel $^{-1}$. Details of the observations can be found in Tables 3 and 4 . The data were collected over four observing runs over a period spanning from 2010 May to 2011 April. Observations were centered around R.A. $=10^{\mathrm{h}} 08^{\mathrm{m}} 29.5$ and decl. $=-0^{\circ} 26^{\mathrm{m}} 45^{\mathrm{s}}$ for Sextans A, and R.A. $=09^{\mathrm{h}} 59^{\mathrm{m}} 26^{\mathrm{s}} .1$ and decl. $=+30^{\circ} 44^{\mathrm{m}} 55^{\mathrm{s} .5}$ for Leo A. Dedicated background control fields were also observed.
Table 2

WHIRC Filter Characteristics

\begin{tabular}{lcc}
\hline \hline Filter & $\lambda_{c}(\mu \mathrm{m})$ & $\Delta \lambda(\mu \mathrm{m})$ \\
\hline$J$ & 1.250 & 0.1619 \\
$H$ & 1.651 & 0.3009 \\
$K_{\mathrm{s}}$ & 2.150 & 0.3430 \\
\hline
\end{tabular}

Table 3

Observation Log for Sextans A

\begin{tabular}{llcccc}
\hline \hline & & & & 3 & Seeing \\
BWHM & \\
Band & $\begin{array}{l}\text { Observation } \\
\text { Date }\end{array}$ & $\begin{array}{c}\text { Interval } \\
(\text { days })^{\mathrm{a}}\end{array}$ & Exp. Time (s) & $\left(^{\prime \prime}\right)$ & Airmass \\
\hline$J$ & 2010 & 0 & 120 & 1.8 & $1.35-1.45$ \\
& May 02 & & & & \\
$J$ & 2011 Jan 23 & 266 & 180 & 1 & $1.70-1.95$ \\
$J$ & 2011 Jan 24 & 267 & 180 & 1.1 & $1.72-2.57$ \\
$J$ & 2011 Apr 15 & 348 & 180 & $\ldots$ & $1.26-1.41$ \\
$H$ & 2011 Jan 24 & $\ldots$ & 180 & 1.6 & $1.30-1.43$ \\
$K_{\mathrm{s}}$ & 2011 Jan 23 & 0 & 60 & 0.7 & $1.25-1.33$ \\
$K_{\mathrm{s}}$ & 2011 Jan 23 & 0 & 80 & 0.7 & $1.48-1.25$ \\
\hline
\end{tabular}

Note.

${ }^{\text {a }}$ Interval is the separation in days between the first observational epoch and latter epochs.

Table 4

Observation Log for Leo A

\begin{tabular}{|c|c|c|c|c|c|}
\hline Band & $\begin{array}{l}\text { Observation } \\
\text { Date }\end{array}$ & $\begin{array}{c}\text { Interval } \\
\text { (days) }\end{array}$ & Exp. Time (s) & $\begin{array}{c}\text { Seeing } \\
\text { FWHM } \\
\left({ }^{\prime \prime}\right)\end{array}$ & Airmass \\
\hline $\bar{J}$ & 2010 Jan 22 & 0 & 180 & 0.8 & $1.19-1.03$ \\
\hline$J$ & 2011 Apr 30 & 463 & 120 & 1.4 & $1.04-1.12$ \\
\hline$H$ & 2011 Jan 24 & $\ldots$ & 180 & 1.6 & $1.01-1.00$ \\
\hline$K_{\mathrm{s}}$ & 2011 Jan 22 & $\ldots$ & 90 & 0.9 & $1.01-1.38$ \\
\hline
\end{tabular}

\subsection{Data Reduction}

The data were reduced in IRAF following the methods detailed in Bruursema et al. (2014) and the steps outlined in the WHIRC Reduction Manual (Joyce 2009). ${ }^{7}$ Briefly, an array linearity correction was performed using the WHIRC task wprep.cl, and the mscred package was used to remove the pupil ghost from the bias-subtracted flat field. The pupil ghost template and pupil removal mask were downloaded from the WIYN-WHIRC NOAO website. ${ }^{8}$ Each individual science image was then sky-subtracted and flat-fielded using a median-filtered sky frame. Finally, bad pixels were removed using standard bad pixel masks and the IRAF fixpix routine.

To determine the astrometry for Sextans A and Leo A, the PyRAF script starfind was used to find the positions of the brightest stars in each field. These stars were then compared to the positions of stars detected in the Two Micron All-sky Survey (2MASS; Cutri \& 2MASS Team 2004; Skrutskie et al. 2006) to find the common stars in the image. Using these 2MASS stars as reference stars, the PyRAF packages xyxymatch and geomap were used to find and correct for position offsets in the frames so that the astrometry of the reduced images to match that of the 2MASS images. Field

\footnotetext{
http://www.noao.edu/kpno/manuals/whirc/

8 https://www.noao.edu/kpno/manuals/whirc/WHIRC.html
} 

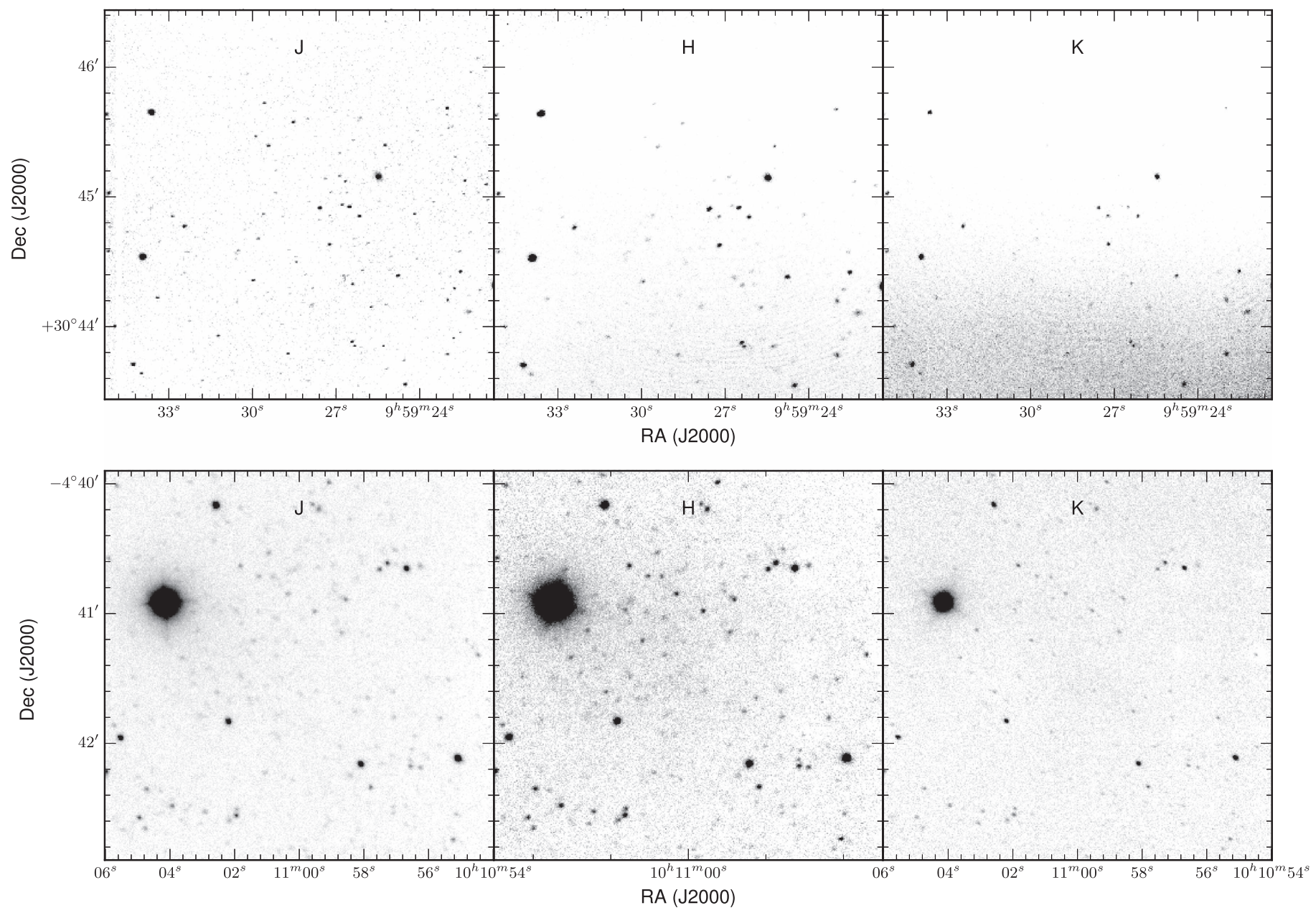

Figure 1. WHIRC $J H K_{s}$ maps of Leo A (top) and Sextans A (bottom). North is at the top, and east is to the left.

distortions in the images were corrected using the geomap and geotrans tasks in IRAF. Final mosaicked images in each filter for the galaxies were produced using MONTAGE. ${ }^{9}$ The WHIRC maps of Sextans A and Leo A are shown in Figure 1.

\subsection{Photometry}

Source extraction, aperture photometry, and related functions were performed with ASTROPY and its recently developed subsidiary package PHOTUTILS ${ }^{10}$ (Astropy Collaboration et al. 2013).

The DAOSTARFINDER function was used to identify point sources in the images. Sharp and round limits were implemented in order to obtain a reliable list of point sources; this cut effectively removes cosmic-ray hits and image artifacts, and minimizes the number of extended sources and resolved background galaxies from the source catalog. Aperture photometry was performed on the resulting source list for each image. The radius of apertures was determined using the predicted full width half maximum of the image resolution, and then adjusted, based on the photometric quality. An outer annulus was used to determine the frame background level, and varied with each image proportionally to the area of the inner aperture.

\footnotetext{
9 http://montage.ipac.caltech.edu/index.html

10 http://photutils.readthedocs.io/en/stable/
}

Preliminary catalog cuts were implemented before flux calibration. Sources with error $\geqslant 65 \%$ or with flux $<0$ were eliminated, as they (a) have a poor signal-to-noise ratio, (b) are background subtraction artifacts, or (c) are non-detections. The remaining sources are considered to be high-confidence point sources with reliable flux measurements.

Detector counts (ADU) of our good sources were calibrated into janskys (Jy) via known 2MASS sources (Cutri \& 2MASS Team 2004; Skrutskie et al. 2006) in the images. With many of our images relying on 4-10 calibration-stars, a systematic conversion error of $2.5 \%$ was added to the calibrated flux to account for scatter in the resulting corrections. However, the relative photometry accuracy within the images is better, as it only depends on the $\mathrm{S} / \mathrm{N}$ of the source and the Poisson noise.

\subsection{Description of the Catalog}

\subsubsection{The WHIRC Point-source Catalog}

The point-source catalog detection statistics are summarized in Table 5. Table 6 lists the 779 sources included in the final point-source catalog for both of the galaxies in our sample. For a source to be included in the full catalog, we require it to be detected in at least two bands or at two epochs with high confidence. We consider a source to be a match if their centroids are within a $1^{\prime \prime}$ radius. If a source was detected over multiple epochs, we present the mean flux and uncertainty for the combined epochs. The WHIRC high-reliability catalog only 
Table 5

Detection Statistics

\begin{tabular}{lcc}
\hline \hline Wavebands & Leo A & Sextans A \\
\hline Total Detected & 287 & 492 \\
$J, H \& K_{s}$ & 38 & 176 \\
$J \& H$ & 98 & 88 \\
$J \& K_{\mathrm{s}}$ & 1 & 24 \\
$H \& K_{\mathrm{s}}$ & 3 & 11 \\
High-reliability Catalog & 140 & 299 \\
WHIRC Variables & 50 & 30 \\
\hline
\end{tabular}

includes sources detected in two or more WHIRC bands. This is less complete than the full catalog but has a more stringent acceptance criteria.

Figure 2 shows a histogram of the number of sources detected in the $J H K_{s}$ photometric bands as a function of magnitude. The faint source detection limit for the $K_{s}$ data of Leo A is almost two magnitudes brighter than for Sextans A due to the high sky background. Although less deep, this data is useful in constraining the SEDs of the brightest sources in the galaxy.

\subsubsection{Ancillary Photometric Data}

In order to fully investigate the nature of the sources in our near-IR catalog; we match our source catalog to all available photometric catalogs for Leo A and Sextans A on a purely positional basis. A matching distance of $3^{\prime \prime}$ was adopted, and if multiple matches occured within a single catalog, only the closest match was kept. The ancillary data was obtained from the Hubble Space Telescope (HST; Bianchi et al. 2012), the Large Binocular Telescope (LBT; Bellazzini et al. 2014), the Subaru Prime Focus Camera (Suprime; Stonkutè et al. 2014), and the Spitzer Space Telescope (Boyer et al. 2009, 2015b, 2015c). These catalogs are not contemporary with the WHIRC data. However, they can aid in source classification and place constraints on the SED from the optical to the mid-IR. Table 7 lists each ancillary catalog, the photometric bands and the number of sources in common with our master catalog.

Most sources $(>90 \%)$ are matched to at least one of these ancillary catalogs. At longer wavelengths, the number of matches decreases, as dust-enshrouded objects are rarer than stellar photospheres. Of the matched sources, nine stars in Sextans A and two stars in Leo A were identified as AGB variable star candidates by Boyer et al. (2015b, 2015c). Our catalog also includes the two known planetary nebulae (Magrini et al. 2003) in these galaxies. WHIRC sources without optical counterparts are rare, however, the large differences in angular resolution and survey depth increases the likelihood for mismatches compared to the WHIRC-IRAC matches. We discuss these objects further in Section 4.

\section{Analysis and Results}

\subsection{Variable Stars}

Evolved stars pulsate, and are variable on timescales of hundreds of days. These pulsations levitate atmospheric material, leading to the formation of dust grains. Thus stars with long-period variations can show a significant IR excess. Our data examines source variability for three epochs, separated by 82,267 , and 363 days for Sextans A, and two epochs separated by 463 days for Leo A. We identify large- amplitude variables from our $J$-band data using the variability index defined by Vijh et al. (2009). This variability index takes into account the error-weighted flux difference between epochs. Figure 3 shows the variability index distribution for Sextans A and Leo A. Following Vijh et al. (2009), sources with $\mid$ Var $\mid>3$ are classified as variable. This conservative threshold (adopted from the LMC's IR stellar populations) corresponds to a $J$-band amplitude of a least $0.25 \mathrm{mag}$ for our sample. We find that 30 variables in Sextans A and 50 variables in Leo A meet this criteria (Table 6).

Our total sample has 11 sources in common with the DUSTiNGS variable catalog. Of these, we identify three as variable using our $J$-band criteria. The majority of these candidate variable stars are bright, with well-characterized errors. Variability indices for fainter stars are less constrained, as small changes in amplitude will be masked by the photometric errors, this effects the identification of the lowest amplitude variable stars in our catalog and those stars significantly obscured by dust (Whitelock et al. 2017). This bias is more pronounced for Sextans A due to its greater distance.

\subsection{Color-Magnitude Diagrams}

In order to classify the variables, we use colors and magnitudes to separate AGB stars from other sources. NearIR colors are particularly useful for roughly discriminating between O- and C-rich AGB stars, and other stellar populations due to their distinctive spectral characteristics. O-rich stars have spectra dominated by $\mathrm{VO}, \mathrm{TiO}$, and $\mathrm{H}_{2} \mathrm{O}$ molecules, while stars with an excess of carbon in the atmosphere are characterized by $\mathrm{CN}$ and $\mathrm{C}_{2}$. The CMDs constructed from the dereddened photometric measurements (see Section 3.4) are shown in Figure 4.

Sources are identified as TP-AGB stars or RSGs candidates if they are brighter than the RGB tip and redder than foreground objects. The empirical relationship determined by Valenti et al. (2004) was used to estimate the near-IR RGB tip in Leo A and Sextans A. Due to the low mass of these systems, identifying other features in the CMDs is challenging. Instead, we adopt the cuts to the $K_{s}$ versus $J-K_{s}$ CMD used by Cioni et al. (2006) and Boyer et al. (2011) to separate the C-AGB and O-AGB stars, and identify dusty AGB stars (which experience molecular blanketing) as those with $J-[8.0] \gtrsim 3.4$ mag or $J-K_{s} \gtrsim 2.2 \mathrm{mag}$ (Blum et al. 2006; Boyer et al. 2011). The location of these cuts, determined from the molecular content and opacity in the stellar photospheres, are shown in Figure 4. In near-IR CMDs, O-AGB stars populate a narrow sequence extending to brighter magnitudes and redder colors, above the tip of the red giant branch (TRGB); carbon stars occupy a broad region to the right of the O-AGB sequence in the form of a red branch (McDonald et al. 2012; Boyer et al. 2015a).

The brightest sources in the CMDs are foreground stars and they have relatively blue colors. Using the TRILEGAL Galactic population synthesis code (Girardi et al. 2005), we expect about 10 foreground stars brighter than $J=21 \mathrm{mag}$ in our Leo A field, and 30 foreground stars in Sextans A, all with $J-H<0.6$ mag.

It should be noted that the variation in metallicity between the galaxies may result in changes to the IR color. The metallicity of the population affects the separation between the parallel lines shown in the top panel of Figure 4. At higher metallicity, O-rich AGB stars occupy a broader range of $J-K$ 
Table 6

Catalog of Point Sources for Leo A and Sextans A

\begin{tabular}{|c|c|c|c|c|c|c|c|c|c|c|c|c|c|c|c|c|c|c|}
\hline Galaxy & ID & R.A. & Decl. & $U$ & $B$ & V & $I$ & $J$ & $\sigma J$ & $H$ & $\sigma H$ & $K$ & $\sigma K$ & {$[3.6]$} & {$[4.5]$} & [5.8] & {$[8.0]$} & Variable \\
\hline$\overline{\text { LeoA }}$ & 0 & 149.8928 & 30.7250 & -99.99 & 23.72 & 22.48 & 21.19 & 19.19 & 0.04 & 19.10 & 0.07 & -99.99 & -99.99 & 19.73 & 19.64 & -99.99 & -99.99 & 0 \\
\hline LeoA & 1 & 149.8593 & 30.7257 & -99.99 & 23.44 & 22.34 & 21.06 & 19.31 & 0.04 & -99.99 & -99.99 & -99.99 & -99.99 & 20.12 & 19.04 & -99.99 & -99.99 & 0 \\
\hline LeoA & 2 & 149.8522 & 30.7259 & -99.99 & 24.57 & 23.98 & -99.99 & 17.07 & 0.08 & 16.87 & 0.09 & 15.89 & 0.10 & 18.06 & 17.76 & 16.30 & -99.99 & 0 \\
\hline
\end{tabular}

u (This table is available in its entirety in machine-readable form.) 


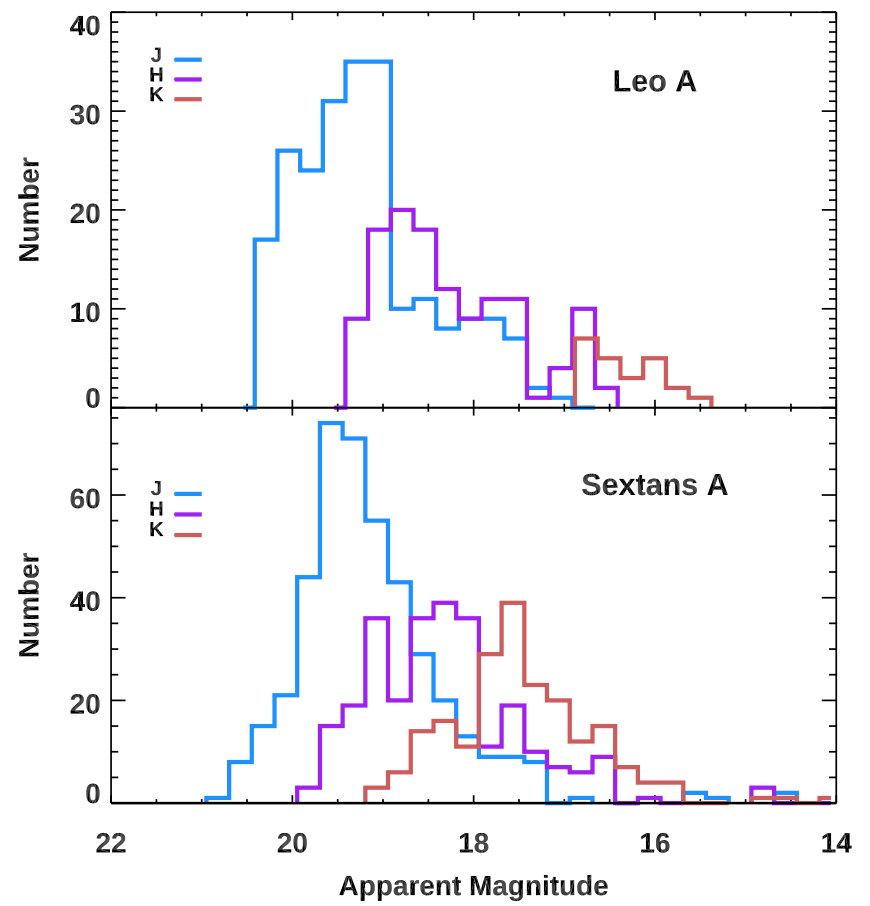

Figure 2. Histogram of the number of sources detected in the three wavebands as a function of magnitude.

Table 7

Number of Matches between the WHIRC Catalogs and Other Photometric Catalogs

\begin{tabular}{|c|c|c|c|c|}
\hline Survey & Filters & Telescope & Leo A & $\overline{\text { Sextans A }}$ \\
\hline $\begin{array}{l}\text { WHIRC—High } \\
\text { Reliability }\end{array}$ & $J H K_{\mathrm{s}}$ & WIYN & 140 & 299 \\
\hline $\begin{array}{l}\text { DUSTiNGS; Boyer et al. } \\
\text { (2015b) }\end{array}$ & {$[3.6],[4.5]$} & Spitzer & 125 & 272 \\
\hline $\begin{array}{l}\text { DUSTiNGS Var; Boyer } \\
\text { et al. }(2015 \mathrm{c})\end{array}$ & {$[3.6],[4.5]$} & Spitzer & 2 & 9 \\
\hline Boyer et al. (2009) & {$[5.8],[8.0]$} & Spitzer & 58 & 120 \\
\hline Bianchi et al. (2012) & $U, B, V, I$ & $H S T$ & $\cdots$ & 177 \\
\hline Bellazzini et al. (2014) & $g, r$ & LBT & $\cdots$ & 292 \\
\hline Stonkutè et al. (2014) & $B, V, I$ & Subaru & 138 & $\cdots$ \\
\hline
\end{tabular}

colors, at low-metallicity their color distribution is narrower. Thus caution is necessary in adopting these color classifications, as the location of the cuts are dependent on the metallicity of the galaxy and the extinction correction, limiting the classification accuracy. As both Leo A and Sextans A have populations formed across a range in metalicities, this may affect the photometric chemical classification of approximately $20 \%$ of the AGB stars in our sample.

For sources without $K_{s}$-band detections, we use the $H$-band data to determine an initial class following the cuts proposed by Sibbons et al. (2012). Stars with $J-H<0.6$ mag are most likely to be main-sequence stars or foreground dwarfs, and stars with $J-H>1.0 \mathrm{mag}$ are classified as carbon stars. For completeness, we also use the $J-[3.6]$ versus [3.6] CMD (i.e., $J-[3.6] \gtrsim 3.1 \mathrm{mag}$ ) suggested by Blum et al. (2006) to separate the O-rich, C-rich, and dusty AGB populations. This is necessary as molecular blanketing due to the fact that dust strongly affects the near-IR spectral region.

For red sources, contamination from unresolved background galaxies may be significant. Focusing on the variable star

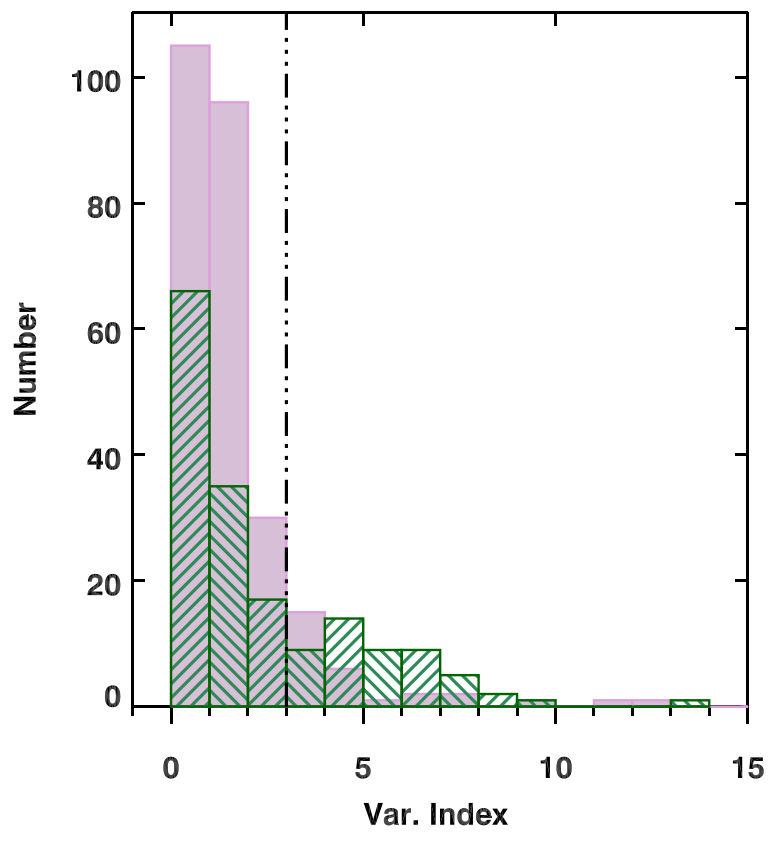

Figure 3. Variability index distribution of all the sources detected in at least two epochs for Leo A (green lines) and Sextans A (pink). We consider a source to be variable if the $J$-band variability index is $>3$ (dashed-dotted line).

population eliminates the majority of this contamination. Conversely, given the SFHs of Sextans A and Leo A, contamination from massive young stellar objects $\left(M>5 M_{\odot}\right)$ is likely very small. The vertical sequence seen in the $J-[3.6]$ CMD is populated by objects with a range in stellar effective temperatures (see Section 3.3), and little IRexcess. In both galaxies, the number of very red objects is modest. The results of the photometric classifications of evolved star candidates are listed in Table 8 .

\subsection{Hertzsprung-Russell Diagrams}

In order to calculate the effective temperature $\left(T_{\text {eff }}\right)$ and bolometric luminosities $\left(M_{\mathrm{bol}}\right)$ of the sources in Sextans A and Leo A, we used the SED-fitting code of McDonald et al. (2012, 2017). This code performs a $\chi^{2}$-minimization between the observed SED (corrected for interstellar reddening) and a grid of BT-SETTL stellar atmosphere models (Allard et al. 2011), which are scaled in flux to derive a bolometric luminosity.

The BT-SETTL spectral models are based on atomic and molecular data; for each galaxy, we used literature values (given in Table 1) for the metallicity, distance, and interstellar reddening. A $\chi^{2}$-minimization is performed, comparing the observed SED to a blackbody function. The derived $T_{\text {eff }}$ and $M_{\text {bol }}$ is then used as a starting point for an iterative $\chi^{2}$-minimization against the interpolated stellar model atmosphere grid. In this way, a precise $( \pm 200 \mathrm{~K})$ fit to the data is achieved. For cases where the SED is not well-sampled, uncertainties are higher. Furthermore, in crowded fields, or areas with background nebulosity, luminosities may be more uncertain.

Figure 5 shows the Hertzsprung-Russell diagram of Leo A and Sextans A we have produced using the derived effective temperatures and bolometric luminosities for all the stars in our field of view. In Leo A, a concentration of stars on a well defined RGB is clearly visible, with the RGB tip at approximately $3800 \mathrm{~K}, 2500 L_{\odot}$. A small AGB population 

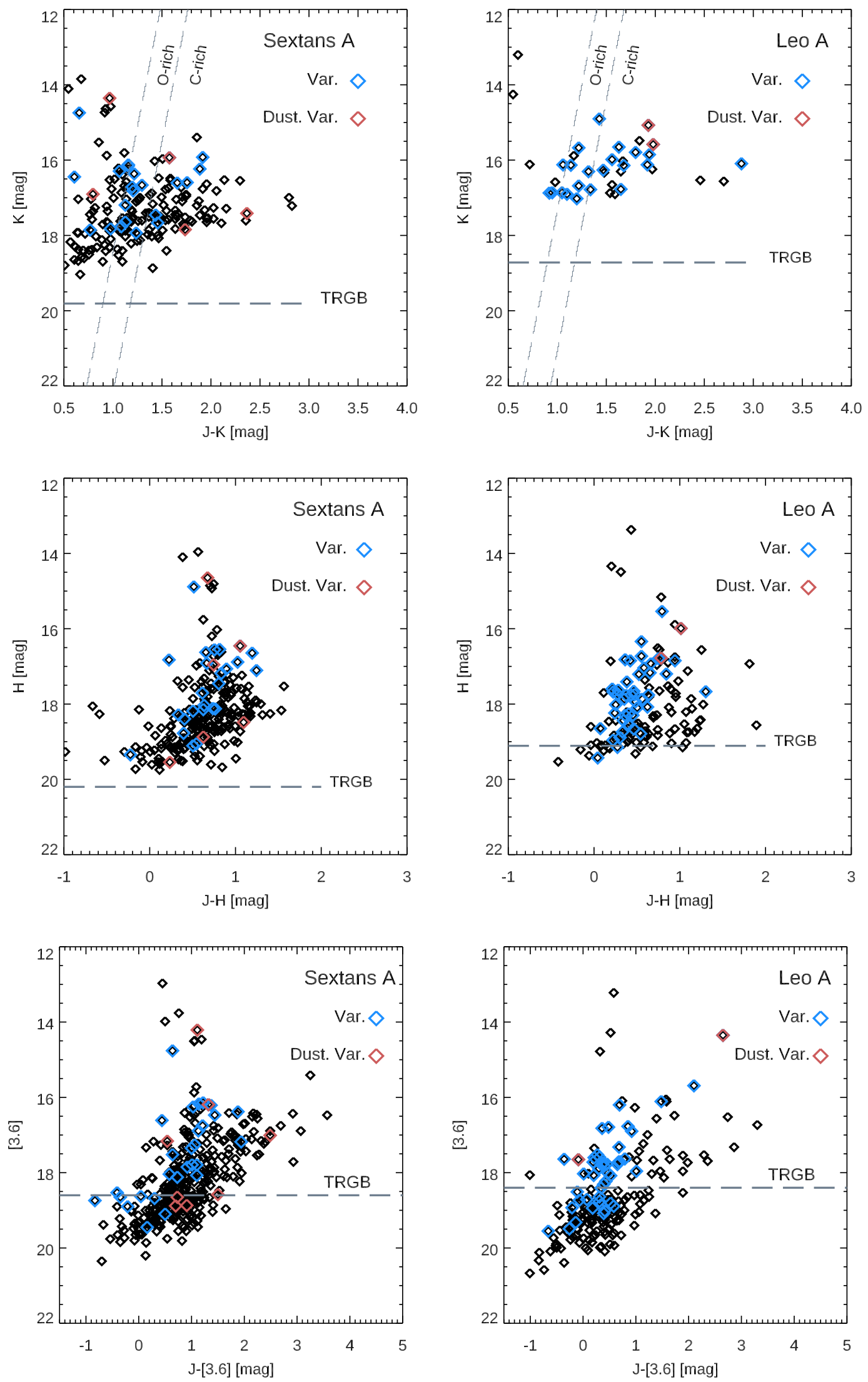

Figure 4. Near- and mid-IR color-magnitude diagrams of stars in Sextans A (left) and Leo A (right), with the average magnitudes of variable star candidates overplotted. Upper panel: $J-K_{\mathrm{S}}$ vs. $K_{s}$ diagram with cuts adopted from Cioni et al. (2006) and Boyer et al. (2011; diagonal dashed lines). Middle panel: $J-H$ vs. $H$ diagram. Lower panel: $J-$ [3.6] vs. [3.6] diagram. In each CMD, the estimated location of the TRGB is shown as a horizontal line. Note that the number of sources in the $J-H$ and the $J-K_{s}$ CMDs are not the same for Leo A, due to the limited sensitivity of the $K_{s}$ data. 
Table 8

Photometric Classification: Evolved Star Candidates

\begin{tabular}{lcc}
\hline \hline Population & Leo A & Sextans A \\
\hline$J<$ TRGB $_{[J]}$ & 57 & 295 \\
O-rich AGB & 10 & 57 \\
C-rich AGB & 18 & 62 \\
Dusty AGB stars & 3 & 18 \\
RSGs & 1 & 8 \\
Foreground & 2 & 3 \\
\hline
\end{tabular}

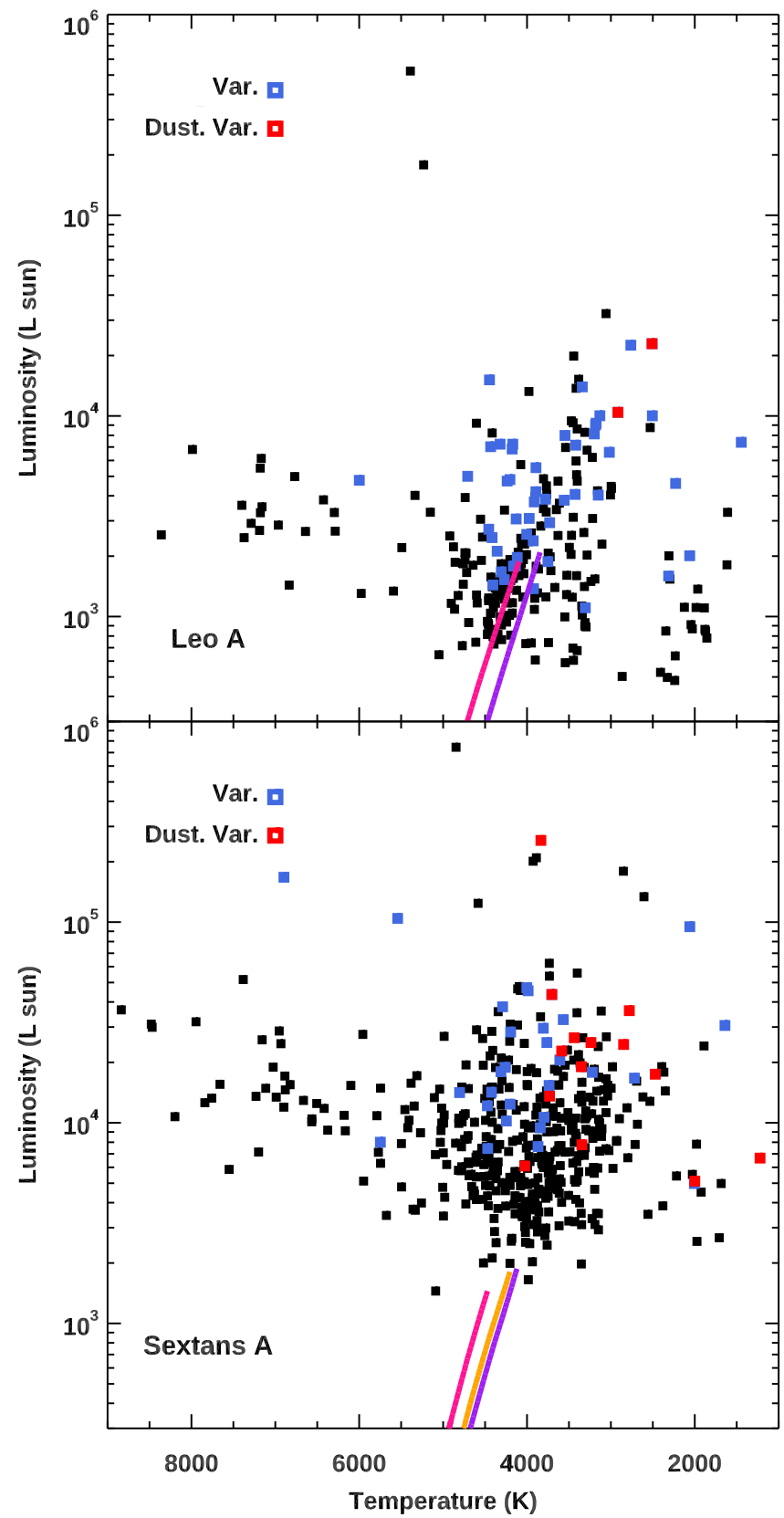

Figure 5. Hertzsprung-Russell diagrams of Leo A (top) and Sextans A (bottom). WHIRC variable stars are shown in blue and DUSTiNGS variables in red. Isochrones for $[\mathrm{Fe} / \mathrm{H}]=-1.4$ and $[\alpha / H]=0.4$, for a $3 \mathrm{~Gy}$ (pink) and $14 \mathrm{~Gy}$ (purple) population, calculated from the Dartmouth models (Dotter et al. 2008) are overlaid on the Leo A data. Isochrones of $[\mathrm{Fe} / \mathrm{H}]=-1.85$ and $[\alpha / \mathrm{H}]=0.4$ for $3 \mathrm{~Gy}$ (pink), $8 \mathrm{~Gy}$ (orange), and $14 \mathrm{~Gy}$ (purple) populations are shown for Sextans A. The top of the isochrones correspond to the RGB tip. appears to continue onward from this, ascending to the top right of the diagram. To the left of the RGB is a population of hotter stars, these are likely to be foreground stars, main-sequence stars, or point sources with insufficient IR data constraining the SED. However, some post-AGB stars, OB stars, Herbig $\mathrm{Ae} / \mathrm{Be}$ stars, and certain kinds of binary stars, could be present in this region at higher luminosities. In Leo A, sources cooler than $3500-3000 \mathrm{~K}$, with luminosities less than $3000 L_{\odot}$ are likely to be background galaxies, although some galaxies may also reside among the RGB and AGB stars.

In Sextans A, there is a large amount of scatter in the $\mathrm{H}-\mathrm{R}$ diagram, and the precise location of the RGB is difficult to define. The scatter is due to a more populated main-sequence and giant branches, combined with worse photometric accuracy. Foreground stars are visible toward the top left of the H-R diagram, while background galaxies lie at cooler temperatures and low luminosities.

In an attempt to separate the RGB and AGB stars from the rest of the population in Sextans A, we overplot our variable star candidates and compare their location to that of the RGB in Leo A. When comparing the two galaxies, it is clear that in Sextans A we are sensitive to only the brightest AGB stars and our $\mathrm{H}-\mathrm{R}$ diagram is therefore incomplete, even at the tip of the RGB, while in Leo A we reach a fainter giant branch population. There may also be a difference between the bright AGB populations. In Sextans A, the scatter in the AGB population may be a result of a higher concentration of carbon stars due to the lower bulk metallicity of the galaxy.

\subsection{Dust-production Rates (DPRs)}

Mass-loss rates for every evolved star in Leo A and Sextans A can be computed by fitting their broadband spectral energy distributions (SEDs) with radiative-transfer models. This allows us to characterize the cumulative dust production in these galaxies, and constrain the evolutionary status and chemical type of the individual stars.

To fit the SEDs of all the stars in our catalog, we use the grid of dusty RSG and AGB models (GRAMS; Sargent et al. 2011; Srinivasan et al. 2011). These models were computed using the 2DUST radiative-transfer code (Ueta \& Meixner 2003), and stellar photosphere models for O-rich (Kučinskas et al. 2005, 2006) and C-rich (Aringer et al. 2009) AGB stars. The O-rich dust is modeled using astronomical silicates (Ossenkopf et al. 1992), and for carbon stars, a mixture of amorphous carbon (amC) and 10\% silicon carbide (SiC) was used.

To model the stars, we use SEDs constructed from the mean flux at each wavelength and we correct for interstellar reddening using the Cardelli et al. (1989) extinction law with $R_{\mathrm{v}}=3.1$ mag. Uncertainties in the optical and near-IR photometric data were increased in quadrature using representative LPV amplitudes, to account for stellar pulsations (see Srinivasan et al. 2016). This allows us to fit SEDs for which a precise phase correction could not be determined.

As the GRAMS models are computed for stars in the LMC, the model fluxes must be scaled before performing the fitting. After correcting for distance and extinction, an optimal scaling factor $(\eta)$ was determined by minimizing the difference between the observed and model fluxes. We then perform a $\chi^{2}$ minimization between the models and the data to obtain the best-fit parameters, which include the DPR, and chemical type. 

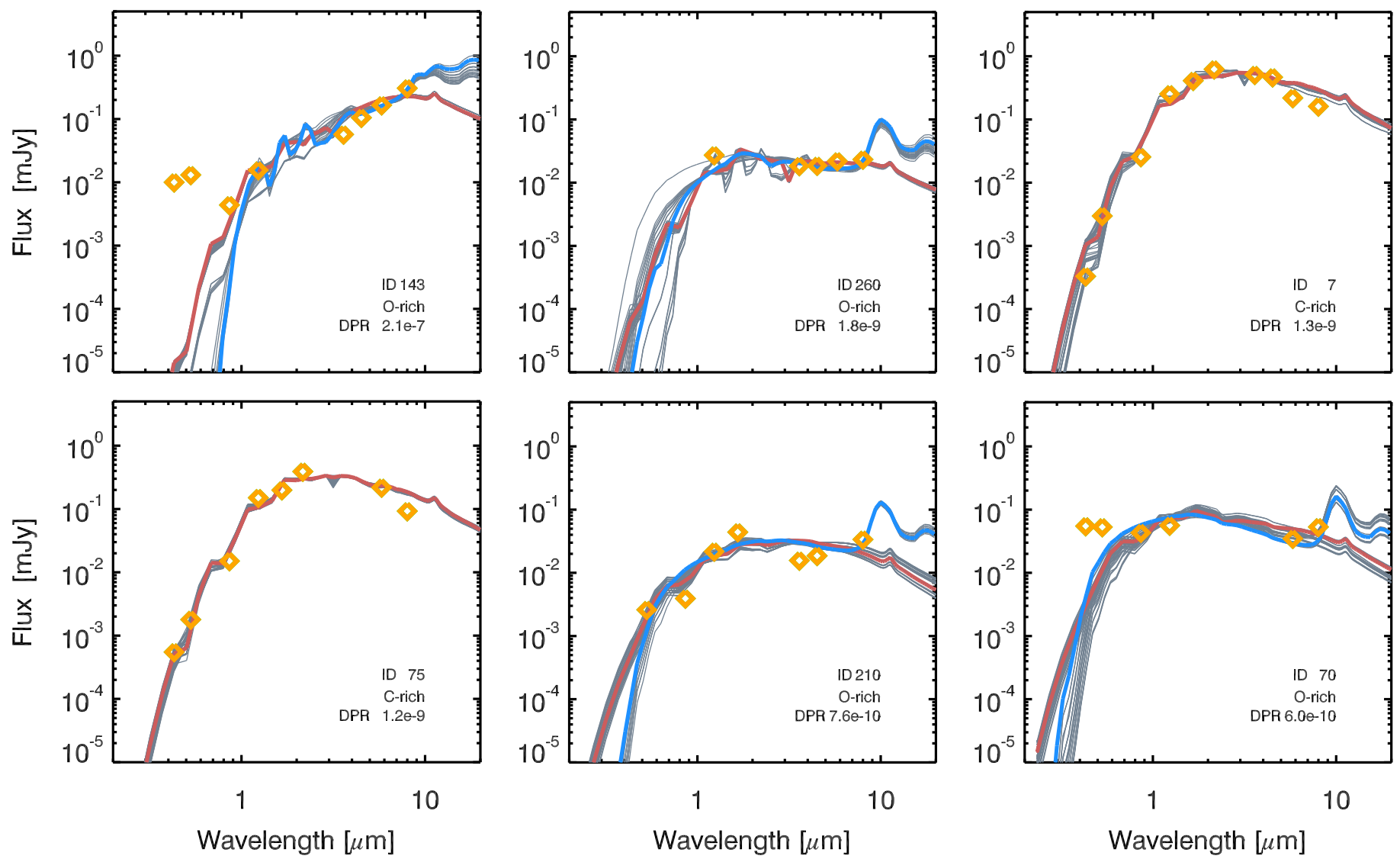

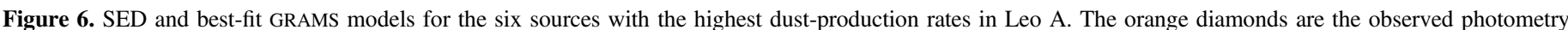

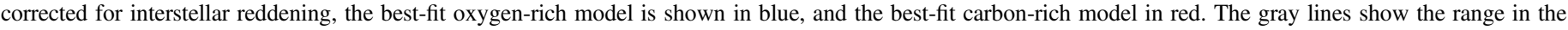

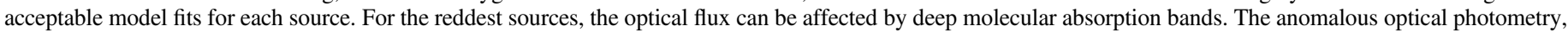

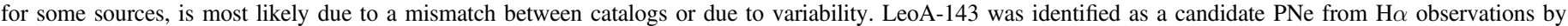
Magrini et al. (2003).

The $\chi^{2}$ value is defined by

$$
\chi_{\nu}^{2}=\frac{1}{N} \sum_{i} \frac{\left(f_{\mathrm{obs}_{i}}-f_{m_{i}}\right)^{2}}{\sigma_{i}^{2}}
$$

where $f_{\mathrm{obs}_{i}}$ and $f_{m_{i}}$ are the observed and model flux in the $i$ th band with error bar $\sigma_{i}$, and $N$ is the total number of data points. As a scaling factor $(\eta)$ has been applied to the models, the model luminosity and wind parameters must also be adjusted. The luminosity scales by $\eta$ and the mass-loss rate by the factor $\sqrt{\eta}$, following Equation (2) of Groenewegen (2006).

To avoid degeneracies in the model fits, we compare the best-fit parameters from the carbon- and oxygen-rich models separately, adopting an approach similar to that of Srinivasan et al. (2016). Here we consider all models that satisfy $\chi^{2}-\chi_{\text {best }}^{2}<2 N_{\text {data }}$, to be acceptable, where $\chi_{\text {best }}^{2}$ is the reduced $\chi^{2}$ value of the best-fitting model and $N_{\text {data }}$ is the number of photometric data points. The uncertainty in each best-fitting parameter is determined from the median absolute deviation of these models. The chemical classification (carbon or oxygen rich) is established from the model with the smallest overall $\chi^{2}$, with a chemical uncertainty determined from the $\chi^{2}$ ratio of the best-fit carbon- and oxygen-rich models.

Figures 6 and 7 show the SEDs and best-fitting models for the six dustiest sources in each galaxy. In both figures, the range of acceptable fits for each source is shown in grey, and, where appropriate, both the best-fit carbon and oxygen-rich model. In instances where only one chemistry provides an acceptable fit to the data, only that chemical type is shown. For the reddest sources, and SEDs with high $\chi^{2}$ values, we carefully inspect the SED and model output to verify the quality of the fitting procedure. In most instances, objects with a high $\chi^{2}$ have SEDs that are inconsistent with a stellar object; they are disjointed, with a poorly defined shape (produced from a mismatch between catalogs) or have too few valid data points. We consider these SEDs to be invalid and discarded their fits from the dust-budget estimates. The results of the model fits for 162 stars in Leo A and 281 stars in Sextans A are listed in Table 9.

\subsubsection{Uncertainties in DPRs}

In Figure 8, we compare the relative uncertainty in the DPR as a function of the DPR for our sample. Sources with DPRs of $\log (\mathrm{DPR})<-11$ have large uncertainties and are consistent with stellar photospheres with no apparent circumstellar excess due to dust. In both Sextans A and Leo A, the majority of the stars detected $(\sim 70 \%)$ are low-DPR sources hence they do not contribute to the dust budget of the galaxy.

When comparing the DPR between galaxies, we have assumed that there is no change in the dust properties with metallicity, and that the wind expansion velocity is constant. A fixed expansion velocity is unlikely if radiation pressure on dust is the primary accelerant of the wind. This has been observed by Marshall et al. (2004), Groenewegen et al. (2016), 

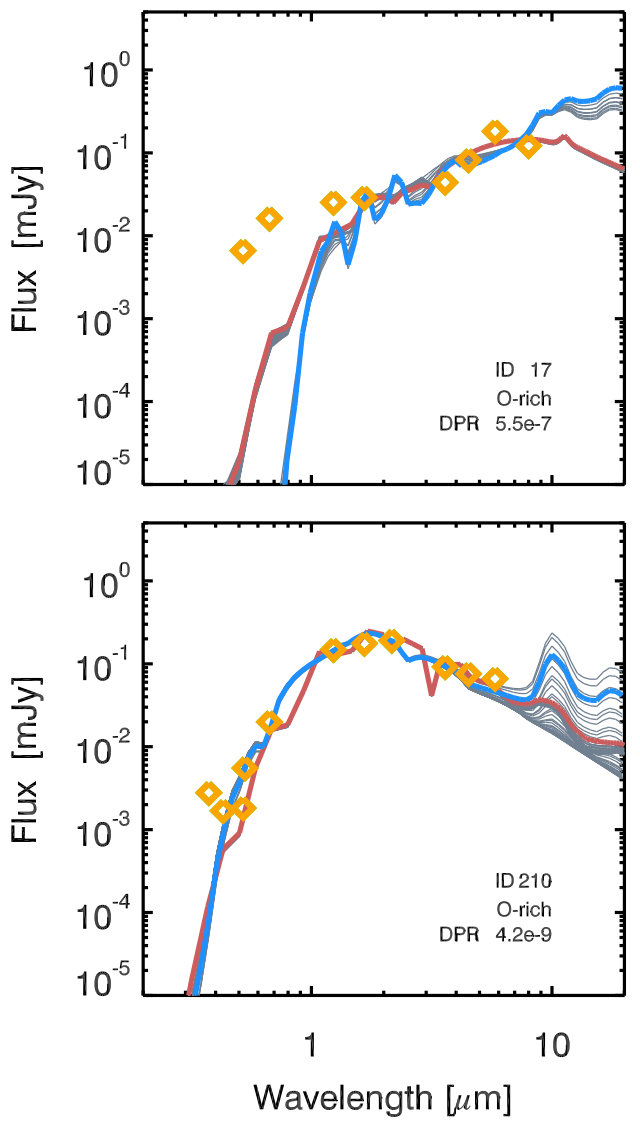
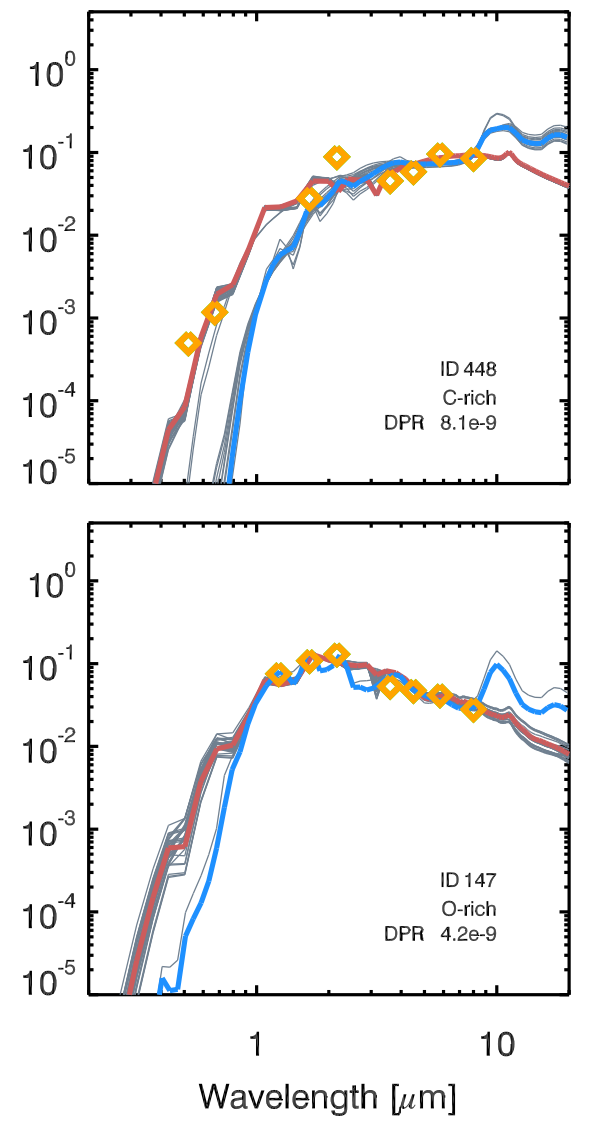
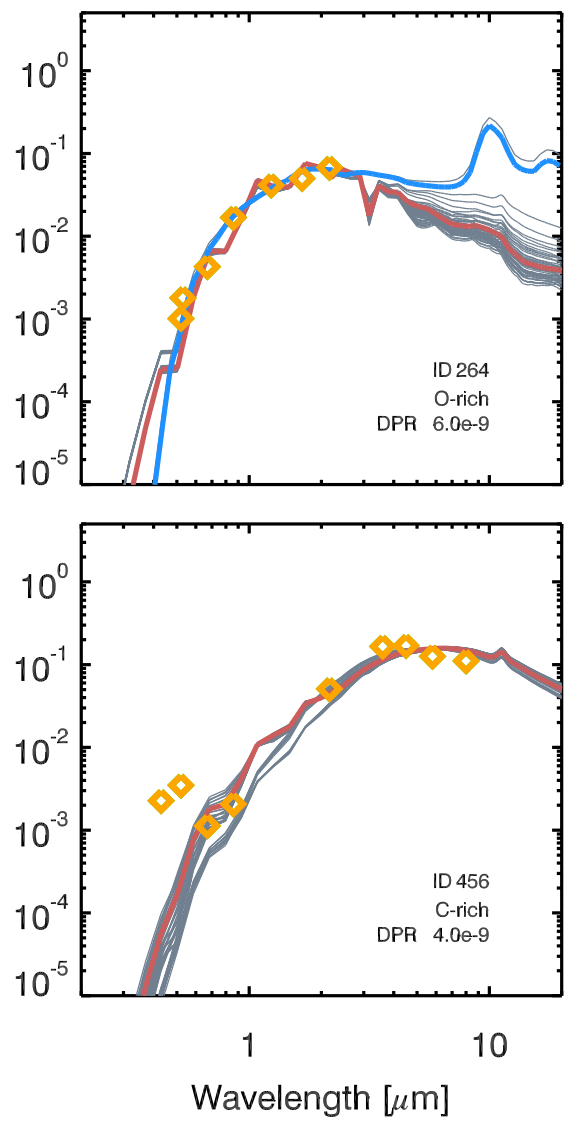

Figure 7. SED and best-fit GRAMS models for the six sources with the highest dust-production rates in Sextans A. Colors are the same as in Figure 6.

and Goldman et al. (2017) who found a decrease in expansion velocity for stars with comparable properties at lower metallicity. Furthermore, Jones et al. (2012, 2014) and McDonald et al. (2010) have shown that the composition of oxygen-rich dust species has a dependence on metallicity. These systematically increase the absolute uncertainty in both the individual DPRs and the global dust injection rates (see Table 2 of McDonald et al. 2011 for a detailed analysis of the inherent uncertainties in DPRs). However, the star-to-star variations are much smaller.

The chemical classification determined from the best-fit GRAMS models also has a large influence on the uncertainty in the DPRs. Low-confidence chemical classifications, where both the $\mathrm{O}$ - and $\mathrm{C}$-rich models produce almost an equally good fit to the data, can have DPR estimates that differ by over an order of magnitude. Figure 9 shows the uncertainty in the chemical classification. The majority of the sources in our sample with $\log (\mathrm{DPR})>-11$ were deemed to have a large chemical uncertainty, with a value close to unity. This is a consequence of a lack of data at mid-IR wavelengths, necessary for breaking the degeneracy in dust chemistry.

\section{Discussion}

\subsection{Late-type Stellar Populations of Leo A and Sextans A}

The differences in the temperature and luminosity distributions between the stellar populations of Leo A and Sextans A can be clearly seen in Figure 10. In Leo A, there is a significant decline in source density toward brighter magnitudes at
$M_{\mathrm{bol}}=-3.6$, which corresponds to the TRGB (Ferraro et al. 2000). Stars below the TRGB are of low mass $\left(M \sim 0.8-2 M_{\odot}\right)$, likely from a population-aged between 1 and 13 Gyr. The depth of the photometry for Sextans A is not sufficient to reach the TRGB; here the luminosity function has a rapid decline toward fainter magnitudes at $M_{\text {bol }}=-4.5$. Our reported AGB population should therefore be considered a lower limit. The discontinuity near $M_{\text {bol }}=-4.5$, may also occur if there was a significant increase in the SFR a few hundred megayears ago.

Leo A has a blue population of giants that have low circumstellar extinction and populate fainter magnitudes, which is consistent with RGB or early-AGB stars. Leo A has only a few red sources that appear to be evolving on the TP-AGB, these stars are bluer than those in Sextans A. The presence of carbon stars in the CMDs provides confirmation of an intermediate-age population in both Leo A and Sextans A. Only intermediate-mass AGB stars ( 2-4 $M_{\odot}$; Marigo \& Girardi 2007; Ventura \& Marigo 2010) can become carbonrich, although the transition luminosity is lower in metal-poor populations. Sextans A appears to have a higher relative fraction of carbon stars compared to Leo A (Table 9), and its evolved stellar population (above the TRGB) is more luminous.

In both galaxies, there are a few stars with bolometric magnitudes brighter than the traditional AGB luminosity limit of $M_{\mathrm{bol}}=-7.1$ (Wood et al. 1983). These bright sources represent a small population of RSGs or hot-bottom-burning AGB stars (with $\left.M>8 M_{\odot}\right)$, whose ages are consistent with a recent increase in the SFR $\sim 1$ Gyr ago (Dolphin et al. 2005; Cole et al. 2007). The 
Table 9

Luminosities and Dust-production Rates of the 443 Evolved Stars with Valid Fits in Leo A and Sextans A

\begin{tabular}{|c|c|c|c|c|c|c|}
\hline Galaxy & WHIRC_ID & $\begin{array}{l}T_{\text {eff }} \\
(\mathrm{k})\end{array}$ & $M_{\mathrm{bol}}$ & $\begin{array}{c}\mathrm{DPR} \\
\left(M_{\odot} \mathrm{yr}^{-1}\right)\end{array}$ & $\begin{array}{c}\sigma \mathrm{DPR} \\
\left(M_{\odot} \mathrm{yr}^{-1}\right)\end{array}$ & GRAMS Chem. \\
\hline LeoA & 3 & 3800 & -4.1 & $5.0 \mathrm{E}-11$ & $1.1 \mathrm{E}-11$ & $\mathrm{C}$ \\
\hline LeoA & 4 & 4100 & -3.7 & $2.3 \mathrm{E}-13$ & $4.8 \mathrm{E}-12$ & $\mathrm{O}$ \\
\hline LeoA & 7 & 2500 & -6.1 & $1.3 \mathrm{E}-09$ & $4.3 \mathrm{E}-10$ & $\mathrm{C}$ \\
\hline LeoA & 9 & 4200 & -3.2 & $1.4 \mathrm{E}-13$ & $2.9 \mathrm{E}-12$ & $\mathrm{O}$ \\
\hline
\end{tabular}

(This table is available in its entirety in machine-readable form.)
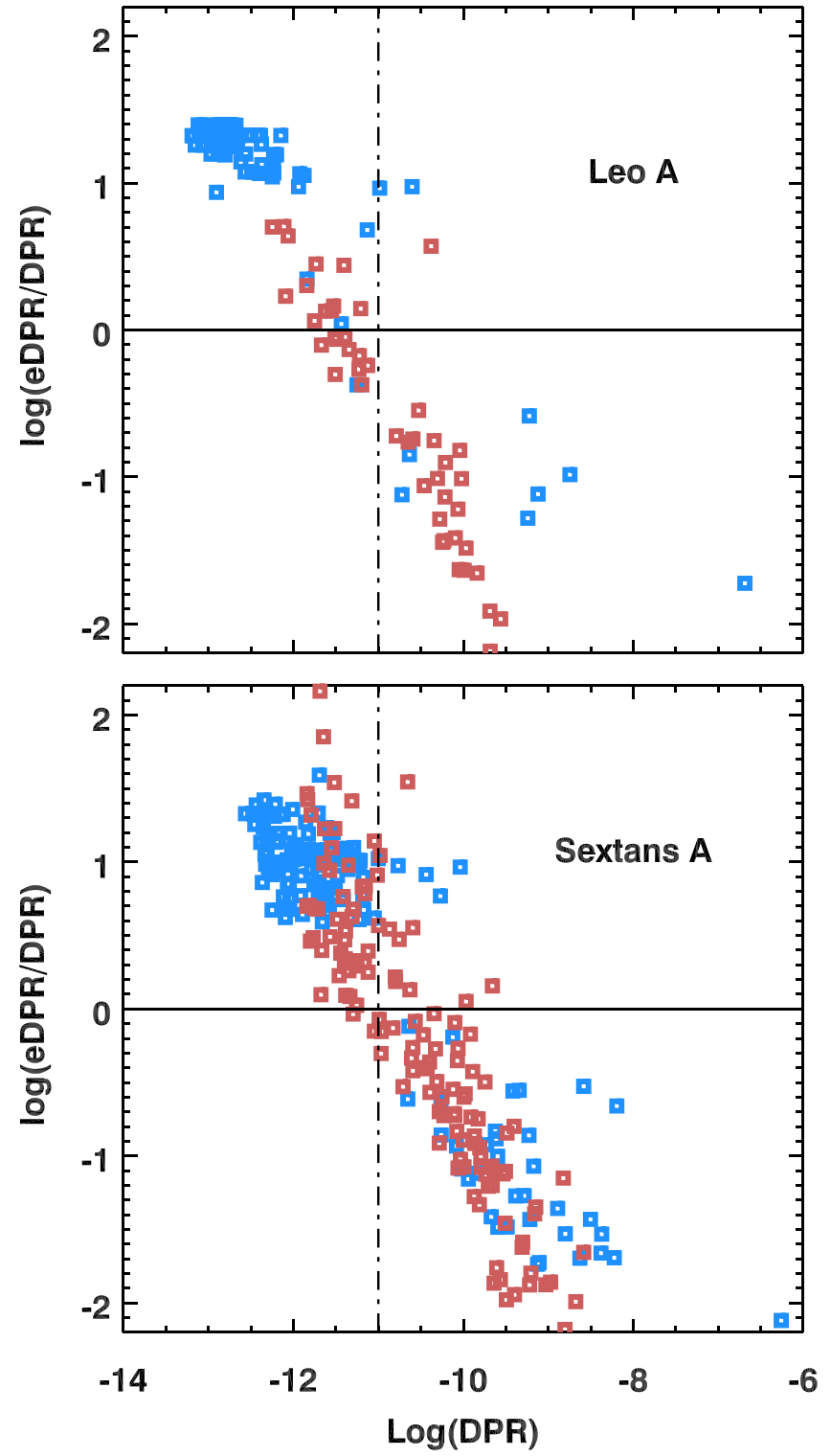

Figure 8. Relative uncertainty in the dust-production rate as a function of the dust-production rate for Leo A (top) and Sextans A (bottom). Oxygen-rich stars are blue and carbon-rich stars are red. Sources to the left of the dashed line are consistent with a mass-loss rate of zero. Stars in the lower-right quadrant are producing dust. Only these sources were used to determine the global dustmass injection into the ISM of each galaxy.

discrepancy in the size of the AGB populations unlikely due to the difference in stellar mass of the host galaxy. More likely it is due to differences in both the mean metallicity and SFH.
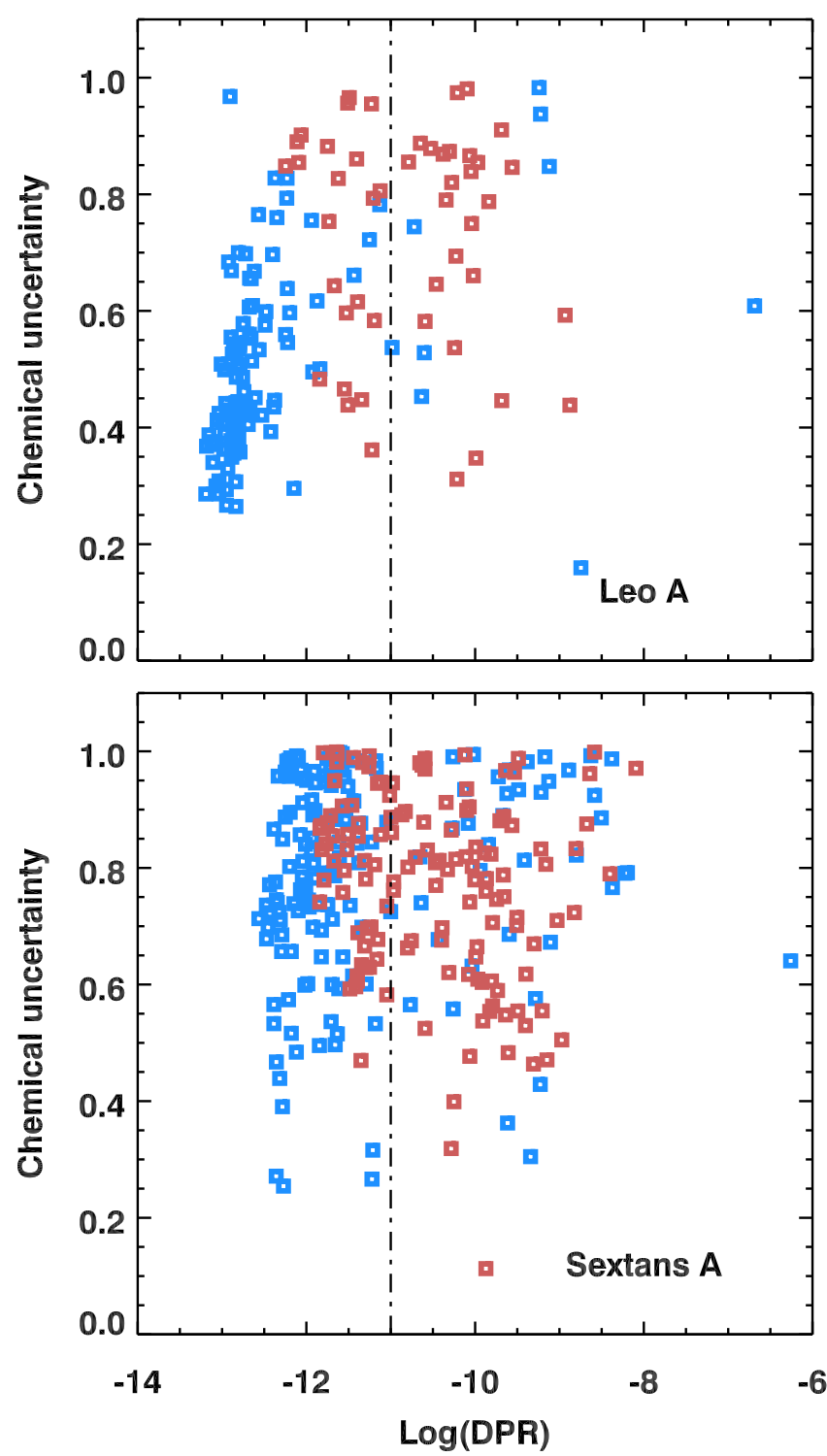

Figure 9. Ratio of the $\chi^{2}$ values for the best-fitting models from the carbonand oxygen-rich model grids for each source. A low value $(<0.7)$ corresponds to a high-confidence chemical classification.

\subsection{Dust Production}

Stars with $\log (\mathrm{DPR})>-11$ have a measurable IR excess due to dust. There are 32 stars in Leo A and 101 stars in Sextans A, which meet this criteria, with a low-moderate relative uncertainty in the DPR. As the uncertainty in the chemical classification is high for most sources (Figure 9), we 

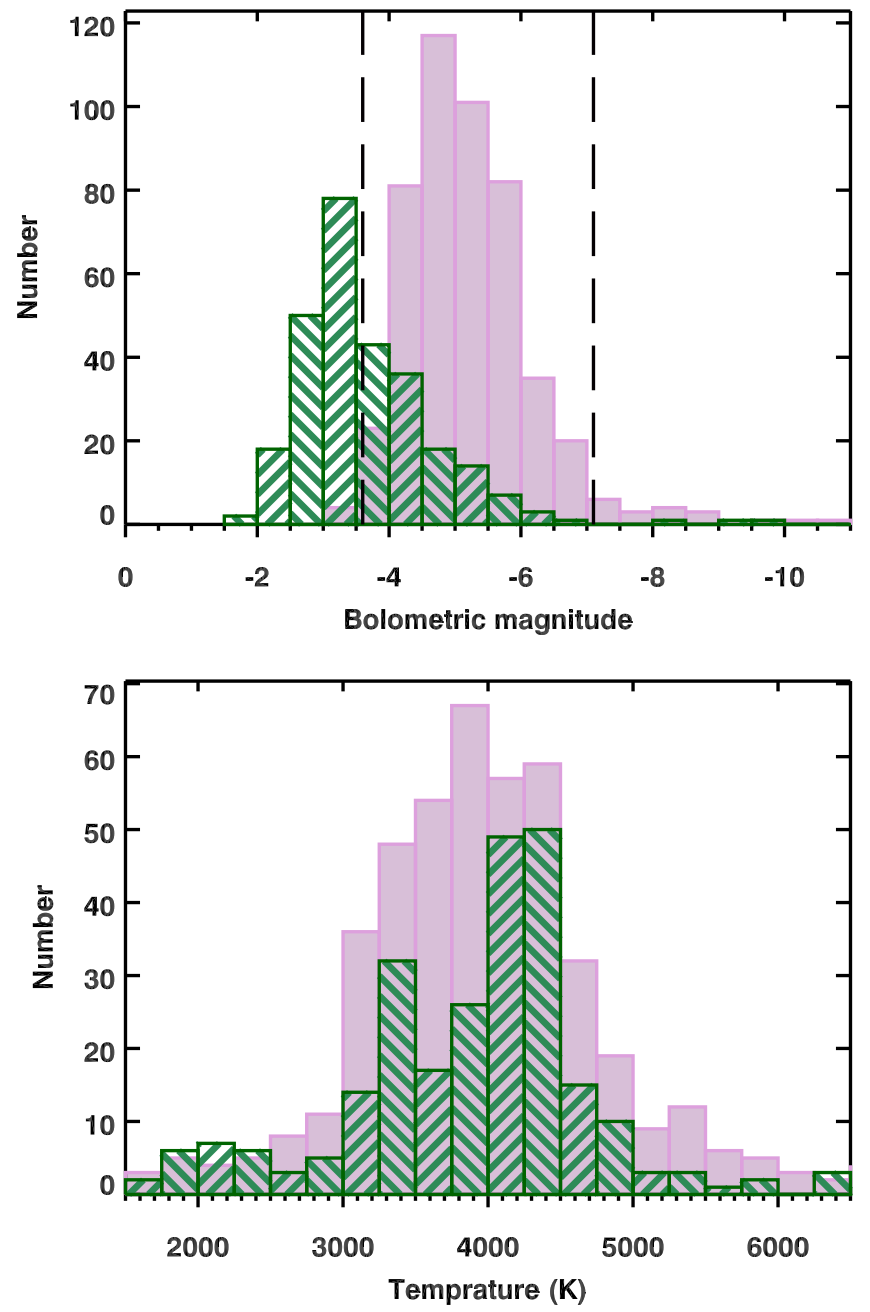

Figure 10. Temperature and luminosity distributions of Leo A (green lines) and Sextans A (pink). The dashed lines in the top panel mark the TRGB at $M_{\mathrm{bol}}=-3.6$ (Ferraro et al. 2000) and the classical AGB limit at $M_{\mathrm{bol}}=-7.1$.

compute four values for the total dust-production rate: (1) using the best-fit models irrespective of dust chemistry; (2) assuming all stars are O-rich; (3) assuming all stars are C-rich; and (4) only including stars identified as variable. Table 10 lists the total DPRs for each galaxy. These estimates exclude any source with a relative uncertainty in the DPR $>0$ (see Figure 8).

In both galaxies, the total dust input into the ISM is dominated by one or two stars. In Leo A, the star with the highest DPR is LeoA-143, with a DPR of $(2.1 \pm 0.1) \times 10^{-7}$ $M_{\odot} \mathrm{yr}^{-1}$. This is two orders of magnitude greater than the second-highest DPR in Leo A. This object has a rising SED and was identified as a candidate $\mathrm{PNe}$ from $\mathrm{H} \alpha$ observations by Magrini et al. (2003), hence we exclude this source from the global dust-budget estimate.

In Sextans A, the two sources with the highest DPRs are IDs 17 and 448 (SSTDUSTG J101059.43-044249.3 and $\mathrm{J} 101058.05-044304.1$, with $(5.5 \pm 0.1) \times 10^{-7} M_{\odot} \mathrm{yr}^{-1}$ and $(8.1 \pm 3.9) \times 10^{-9} M_{\odot} \mathrm{yr}^{-1}$, respectively). These also have rising SEDs; however, both of these sources have been identified as variable $\mathrm{x}$-AGB candidates by DUSTiNGS (Boyer et al. 2015c). Our fitting routine classified SextansA-17 as an oxygen-rich AGB-star; it is responsible for $89 \%$ of the global dust production of Sextans A. If this source is instead carbon rich, then its DPR is significantly lower: $(1.2 \pm 0.3) \times$ $10^{-8} M_{\odot} \mathrm{yr}^{-1}$. If SextansA-17 is confirmed to be an oxygenrich AGB star, then it conclusively shows that individual metalpoor $([\mathrm{Fe} / \mathrm{H}] \sim-1.85$ to -1.40$)$ evolved stars can produce significant amounts of dust, and contribute substantially to the enrichment of the ISM in their host system.

Figure 11 compares the DPR to a source's bolometric magnitude. For the stars with a circumstellar excess, $\log (\mathrm{DPR})>-11$, no trend is seen between mass-loss rate and luminosity. This is surprising, as both luminosity and DPR should increase during the stars' evolution on the AGB, as expected from most mass-loss prescriptions (e.g., Vassiliadis \& Wood 1993; Bloecker 1995). A similar effect was seen in the LMC by Riebel et al. (2012). Three luminous carbon stars are observed in Sextans A, which are in excess of the maximum AGB luminosity limit; however, all three have low-confidence chemical classifications. If they are indeed carbon-rich they are unlikely to be AGB stars associated with the galaxy and are presumably foreground stars. All stars brighter than $M_{\mathrm{bol}}=-9.0$ are considered foreground sources, and are excluded from global DPR estimates.

The most extreme AGB stars, producing the largest amount of dust are near to the end of their AGB evolution. The RGBtip is at approximately $M_{\text {bol }} \sim-3.6 \mathrm{mag}$, TP-AGB stars should all be brighter than this limit (e.g., Rosenfield et al. 2014). Consequently, our data represents a near-census of the TP-AGB stars in Leo A and we can separate the dustproducing AGB stars from the larger RGB population. In Sextans A, we do not reach the RGB tip, as we are limited by sensitivity, so our dust-production value for this galaxy should be considered a lower limit.

\subsubsection{Comparison of Global Dust-production}

Sextans A is at a lower metallicity than Leo A, yet the cumulative DPR of Sextans A is significantly higher than for Leo A, even when accounting for the difference in stellar mass between the galaxies. This result is consistent with Boyer et al. (2015c), who estimated the rate of dust production by AGB stars in Leo A to be $5.2 \times 10^{-9} M_{\odot} \mathrm{yr}^{-1}$, and Sextans A to be $9.3 \times 10^{-8} M_{\odot} \mathrm{yr}^{-1}$. However, our results disagree with the trends observed from the evolved-star dust budgets of the Magellanic Clouds (Srinivasan et al. 2016). Here the SMC is observed to have consistently lower DPRs than the LMC; this is thought to be due to the lower mean metallicity of the galaxy.

As the LMC and SMC are closer, have well-characterized evolved star populations and have SEDs that are better sampled at IR wavelengths than Leo A and Sextans A, the integrated mass-loss of the Magellanic Clouds is less likely to be affected by stochastics from a few rare objects that are experiencing strong mass loss, contamination from other source types, and by systematic errors in the SED fitting.

The main source of uncertainty in our dust-budget estimates are due to the ambiguous evolutionary status of a small number of sources, rather than the chemical uncertainty of the dust. This is because the DPRs determined with carbon-star models are, on average, higher than with oxygen-rich models, for individual stars with a small IR-excess. Conversely, the oxygen-rich models return higher DPRs than the carbon-rich models for redder stars.

For both galaxies the median DPRs calculated by Boyer et al. (2015c) is about an order of magnitude higher than our 
Table 10

The Global Dust-production Rates for Leo A and Sextans A

\begin{tabular}{lccc}
\hline \hline \multirow{2}{*}{ Population } & \multicolumn{2}{c}{ Leo A } & \multicolumn{2}{c}{ Sextans A } \\
\cline { 2 - 5 } & $\begin{array}{c}\text { Total DPR } \\
\left(M_{\odot} \mathrm{yr}^{-1}\right)\end{array}$ & $\begin{array}{c}\text { Median DPR } \\
\left(M_{\odot} \mathrm{yr}^{-1}\right)\end{array}$ & $\begin{array}{c}\text { Total DPR } \\
\left(M_{\odot} \mathrm{yr}^{-1}\right)\end{array}$ \\
\hline Best-fitting Chemical Type & $(8.2 \pm 1.8) \times 10^{-9}$ & $8.1 \times 10^{-11}$ & $(6.2 \pm 0.2) \times 10^{-7}$ \\
If all O-rich & $(6.5 \pm 2.8) \times 10^{-9}$ & $6.2 \times 10^{-10}$ & $(6.3 \pm 1.5) \times 10^{-7}$ \\
If all C-rich & $(5.0 \pm 1.5) \times 10^{-9}$ & $8.1 \times 10^{-11}$ & $\left.(4.4 \pm 1.3) \times 10^{-8}\right)$ \\
Variables Only & $(2.9 \pm 0.8) \times 10^{-9}$ & $2.1 \times 10^{-10}$ & $(5.7 \pm 0.1) \times 10^{-7}$ \\
\hline
\end{tabular}

Note. Best-fitting chemical type: 25 C-rich and 6 O-rich in Leo A, and 68 C-rich and 33 O-rich in Sextans A. The total DPR in Sextans A should be considered a lower limit, as we do not detect AGB stars to the tip of the RGB.
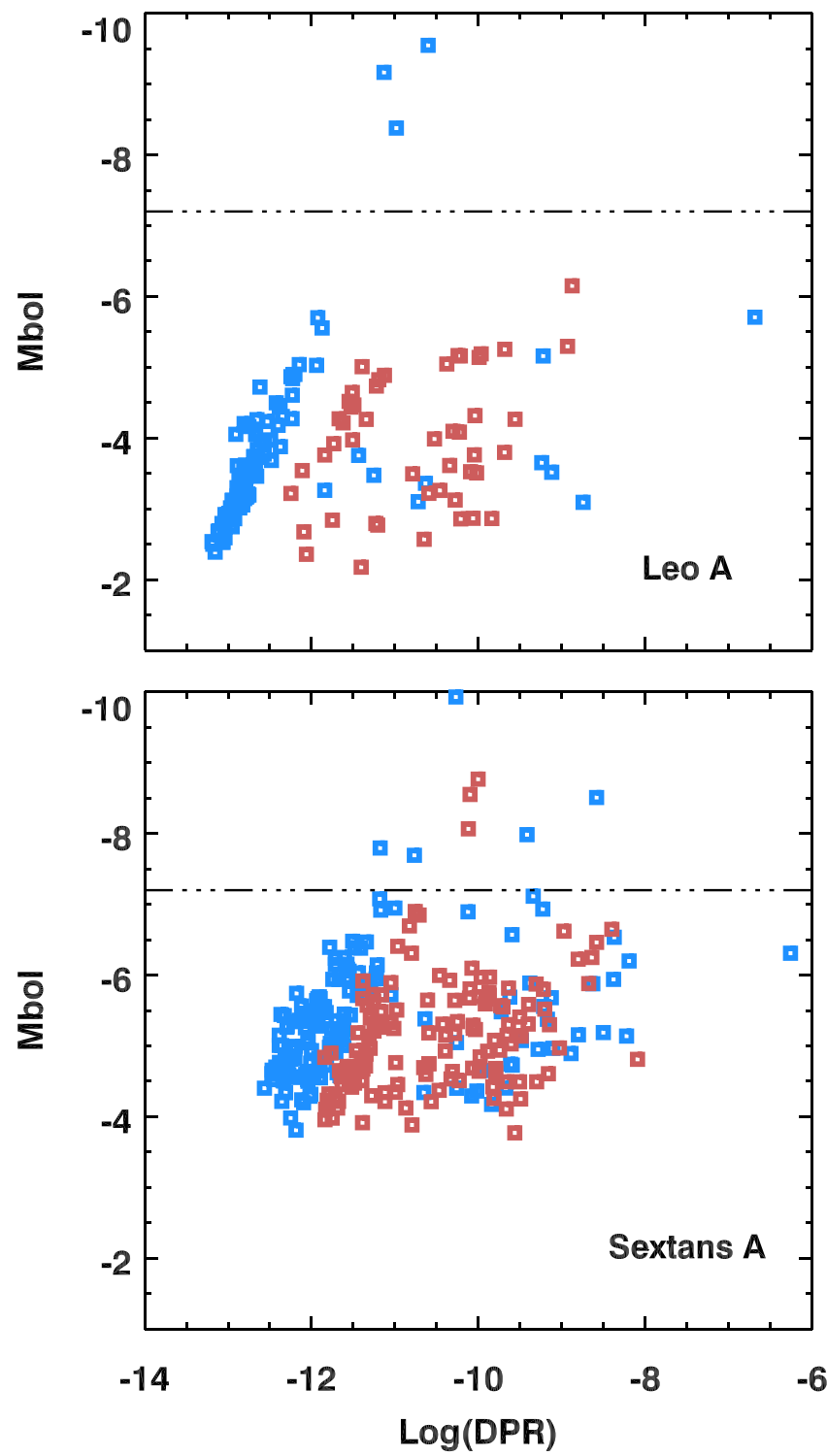

Figure 11. Bolometric magnitude as a function of the dust-production rate for Leo A (top) and Sextans A (bottom). The horizontal line marks the classical AGB luminosity limit, all other symbols are the same as in Figure 8.

estimates. This is expected as the Boyer et al. (2015c) values only include variable stars redder than [3.6]-[4.5] $>0.1$. Whereas our calculations include all stars with a circumstellar excess, the majority of which are producing dust at low rates and are not in the super-wind phase of evolution. However, the same general trend is seen with the median dust output showing no effects due to metallicity.

Approximately a quarter of all the stars that are producing dust at rates of $\log (\mathrm{DPR})>-11$ are best fit with oxygen-rich models. This is unexpected since the abundance of silicon atoms at these metallicities is thought to be too low to form silicate dust grains (Di Criscienzo et al. 2013; Zhukovska \& Henning 2013; Schneider et al. 2014), whereas carbon stars can still produce dust, due to the progressive enrichment of carbon atoms in the surface regions via dredged up from the nuclosynthesis in the core (Karakas et al. 2009). Thus we would expect to see more carbon-rich dust at lower metallicities, and almost no silicate dust.

As noted earlier, the constraints on the dust chemistry are poor; however, if these AGB stars are forming silicate dust, then this suggests that these stars may be more metal-rich, and hence younger and of higher mass, than the rest of the AGB population in Leo A and Sextans A. Alternatively, this dust might be nucleating in a circumbinary disk, or that silicate dust may form via an alternate reaction mechanism to metal-rich stars, from silicon atoms synthesized at the end of the RSG evolutionary phase (Weaver \& Woosley 1980). The mass-loss process, in these metal-poor stars, may also be fundamentally different to the pulsation-enhanced radiation-driven wind (e.g., Höfner 2008) we might naively expect. To verify these results, observations with JWST in the mid-IR would place significant constraints on both the dust chemistry and the stellar type.

\section{Summary}

$J H K_{s}$ images taken with the WIYN telescope at Kitt Peak are used to investigate the evolved star content of the Local Group galaxies Leo A and Sextans A. These observations have allowed us to characterize the near-IR stellar populations using variability, and color-magnitude diagrams to identify evolved stars. In Sextans A, only stars above the TRGB are detected. Matching our data to broadband optical and mid-IR photometry, we determine luminosities, temperatures, and DPRs for each star via SED fitting and radiative-transfer modeling. We identify 32 stars in Leo A and 101 stars in Sextans A with $\log (\mathrm{DPR})>-11$, confirming that metal-poor stars can form substantial amounts of dust. Their dust-mass injection rate into the ISM of Leo A and Sextans A was determined to be $(8.2 \pm 1.8) \times 10^{-9} M_{\odot} \mathrm{yr}^{-1}$ and $(6.2 \pm 0.2) \times 10^{-7} M_{\odot} \mathrm{yr}^{-1}$, respectively. The majority of the dust in each galaxy is produced by a small number of evolved sources. We also find tentative evidence for O-rich dust formation at low metallicity. To confirm the nature of these objects additional highresolution imaging or spectroscopic data are necessary. 
Jones and Meixner acknowledge financial support from NASA grant NNX14AN06G. I.M. acknowledges support from the UK Science and Technology Facility Council under grant ST/L000768/1. This research relied on the following resources: NASA's Astrophysics Data System and the SIMBAD and VizieR databases, operated at the Centre de Données astronomiques de Strasbourg. This research made use of Astropy, a community-developed core Python package for Astronomy (Astropy Collaboration et al. 2013).

Facility: WIYN:0.9 m WHIRC.

\section{ORCID iDs}

Olivia C. Jones (1D https://orcid.org/0000-0003-4870-5547

Matthew T. Maclay (i) https://orcid.org/0000-0001-7860-0055

Martha L. Boyer (ib https://orcid.org/0000-0003-4850-9589

\section{References}

Allard, F., Homeier, D., \& Freytag, B. 2011, in ASP Conf. Ser. 448, XVI Cambridge Workshop on Cool Stars, Stellar Systems, and the Sun, ed. C. Johns-Krull, M. K. Browning, \& A. A. West (San Francisco, CA: ASP), 91

Aringer, B., Girardi, L., Nowotny, W., Marigo, P., \& Lederer, M. T. 2009, A\&A, 503, 913

Astropy Collaboration, Robitaille, T. P., Tollerud, E. J., et al. 2013, A\&A, 558, A33

Bellazzini, M., Beccari, G., Fraternali, F., et al. 2014, A\&A, 566, A44

Bernard, E. J., Monelli, M., Gallart, C., et al. 2013, MNRAS, 432, 3047

Bianchi, L., Efremova, B., Hodge, P., Massey, P., \& Olsen, K. A. G. 2012, AJ, 143, 74

Bloecker, T. 1995, A\&A, 297, 727

Blum, R. D., Mould, J. R., Olsen, K. A., et al. 2006, AJ, 132, 2034

Boyer, M. L., McDonald, I., Srinivasan, S., et al. 2015a, ApJ, 810, 116

Boyer, M. L., McQuinn, K. B. W., Barmby, P., et al. 2015b, ApJS, 216, 10

Boyer, M. L., McQuinn, K. B. W., Barmby, P., et al. 2015c, ApJ, 800, 51

Boyer, M. L., Skillman, E. D., van Loon, J. T., Gehrz, R. D., \& Woodward, C. E. 2009, ApJ, 697, 1993

Boyer, M. L., Srinivasan, S., van Loon, J. T., et al. 2011, AJ, 142, 103

Brown, W. R., Geller, M. J., Kenyon, S. J., \& Kurtz, M. J. 2007, ApJ, 666, 231

Bruursema, J., Meixner, M., Long, K. S., \& Otsuka, M. 2014, AJ, 148, 41

Camacho, I., Garcia, M., Herrero, A., \& Simón-Díaz, S. 2016, A\&A, 585, A82

Cardelli, J. A., Clayton, G. C., \& Mathis, J. S. 1989, ApJ, 345, 245

Cioni, M.-R. L., Girardi, L., Marigo, P., \& Habing, H. J. 2006, A\&A, 452, 195

Cole, A. A., Skillman, E. D., Tolstoy, E., et al. 2007, ApJL, 659, L17

Cutri, R. M. \& 2MASS Team 2004, BAAS, 36, 1487

Di Criscienzo, M., Dell'Agli, F., Ventura, P., et al. 2013, MNRAS, 433, 313

Dohm-Palmer, R. C., Skillman, E. D., Mateo, M., et al. 2002, AJ, 123, 813

Dolphin, A. E., Saha, A., Claver, J., et al. 2002, AJ, 123, 3154

Dolphin, A. E., Saha, A., Skillman, E. D., et al. 2003a, AJ, 125, 1261

Dolphin, A. E., Saha, A., Skillman, E. D., et al. 2003b, AJ, 126, 187

Dolphin, A. E., Weisz, D. R., Skillman, E. D., \& Holtzman, J. A. 2005, arXiv: astro-ph/0506430

Dotter, A., Chaboyer, B., Jevremović, D., et al. 2008, ApJS, 178, 89

Ferraro, F. R., Montegriffo, P., Origlia, L., \& Fusi Pecci, F. 2000, AJ, 119,1282
Ferrarotti, A. S., \& Gail, H. 2001, A\&A, 371, 133

Girardi, L., Groenewegen, M. A. T., Hatziminaoglou, E., \& da Costa, L. 2005, A\&A, 436, 895

Girardi, L., \& Marigo, P. 2007, A\&A, 462, 237

Goldman, S. R., van Loon, J. T., Zijlstra, A. A., et al. 2017, MNRAS, 465, 403 Groenewegen, M. A. T. 2006, A\&A, 448, 181

Groenewegen, M. A. T., Vlemmings, W. H. T., Marigo, P., et al. 2016, A\&A, 596, A50

Höfner, S. 2008, A\&A, 491, L1

Iben, I., Jr., \& Renzini, A. 1983, ARA\&A, 21, 271

Jones, O. C., Kemper, F., Sargent, B. A., et al. 2012, MNRAS, 427, 3209

Jones, O. C., Kemper, F., Srinivasan, S., et al. 2014, MNRAS, 440, 631

Joyce, D. 2014, WIYN High-Resolution Infrared Camera (WHIRC): Quick Guide to Data Reduction v2.0 (Tucson, AZ: NOAO), https://www.noao. edu/kpno/manuals/whirc/WHIRC_Datared_v2.0_140626.pdf

Karakas, A. I., van Raai, M. A., Lugaro, M., Sterling, N. C., \& Dinerstein, H. L. 2009, ApJ, 690, 1130

Kirby, E. N., Rizzi, L., Held, E. V., et al. 2017, ApJ, 834, 9

Kučinskas, A., Hauschildt, P. H., Brott, I., et al. 2006, A\&A, 452, 1021

Kučinskas, A., Hauschildt, P. H., Ludwig, H.-G., et al. 2005, A\&A, 442, 281

Magrini, L., Corradi, R. L. M., Greimel, R., et al. 2003, A\&A, 407, 51

Magrini, L., Leisy, P., Corradi, R. L. M., et al. 2005, A\&A, 443, 115

Maraston, C., Daddi, E., Renzini, A., et al. 2006, ApJ, 652, 85

Marigo, P., \& Girardi, L. 2007, A\&A, 469, 239

Marshall, J. R., van Loon, J. T., Matsuura, M., et al. 2004, MNRAS, 355, 1348

McConnachie, A. W. 2012, AJ, 144, 4

McDonald, I., Sloan, G. C., Zijlstra, A. A., et al. 2010, ApJL, 717, L92

McDonald, I., van Loon, J. T., Sloan, G. C., et al. 2011, MNRAS, 417, 20

McDonald, I., White, J. R., Zijlstra, A. A., et al. 2012, MNRAS, 427, 2647

McDonald, I., Zijlstra, A. A., \& Watson, R. A. 2017, MNRAS, 471, 770

McQuinn, K. B. W., Boyer, M. L., Mitchell, M. B., et al. 2017, ApJ, 834, 78

Meixner, M., Galliano, F., Hony, S., et al. 2010, A\&A, 518, L71

Melbourne, J., Williams, B. F., Dalcanton, J. J., et al. 2012, ApJ, 748, 47

Ossenkopf, V., Henning, T., \& Mathis, J. S. 1992, A\&A, 261, 567

Riebel, D., Srinivasan, S., Sargent, B., \& Meixner, M. 2012, ApJ, 753, 71

Rosenfield, P., Marigo, P., Girardi, L., et al. 2014, ApJ, 790, 22

Sargent, B. A., Srinivasan, S., \& Meixner, M. 2011, ApJ, 728, 93

Schneider, R., Valiante, R., Ventura, P., et al. 2014, MNRAS, 442, 1440

Sibbons, L. F., Ryan, S. G., Cioni, M.-R. L., Irwin, M., \& Napiwotzki, R. 2012, A\&A, 540, A135

Skrutskie, M. F., Cutri, R. M., Stiening, R., et al. 2006, AJ, 131, 1163

Srinivasan, S., Boyer, M. L., Kemper, F., et al. 2016, MNRAS, 457, 2814

Srinivasan, S., Sargent, B. A., \& Meixner, M. 2011, A\&A, 532, A54

Stonkute, R., Arimoto, N., Hasegawa, T., et al. 2014, ApJS, 214, 19

Strobel, N. V., Hodge, P., \& Kennicutt, R. C., Jr. 1991, ApJ, 383, 148

Ueta, T., \& Meixner, M. 2003, ApJ, 586, 1338

Valenti, E., Ferraro, F. R., \& Origlia, L. 2004, MNRAS, 354, 815

van den Bergh, S. 2000, The Galaxies of the Local Group (Cambridge: Cambridge Univ. Press)

Vassiliadis, E., \& Wood, P. R. 1993, ApJ, 413, 641

Ventura, P., \& Marigo, P. 2010, MNRAS, 408, 2476

Vijh, U. P., Meixner, M., Babler, B., et al. 2009, AJ, 137, 3139

Weaver, T. A., \& Woosley, S. E. 1980, NYASA, 336, 335

Weisz, D. R., Dolphin, A. E., Skillman, E. D., et al. 2014, ApJ, 789, 147

Whitelock, P. A., Kasliwal, M., \& Boyer, M. 2017, Euro. Phy. J. Web Conf., 152, 01009

Wood, P. R., Bessell, M. S., \& Fox, M. W. 1983, ApJ, 272, 99

Young, L. M., \& Lo, K. Y. 1996, ApJ, 462, 203

Zhukovska, S., \& Henning, T. 2013, A\&A, 555, A99 